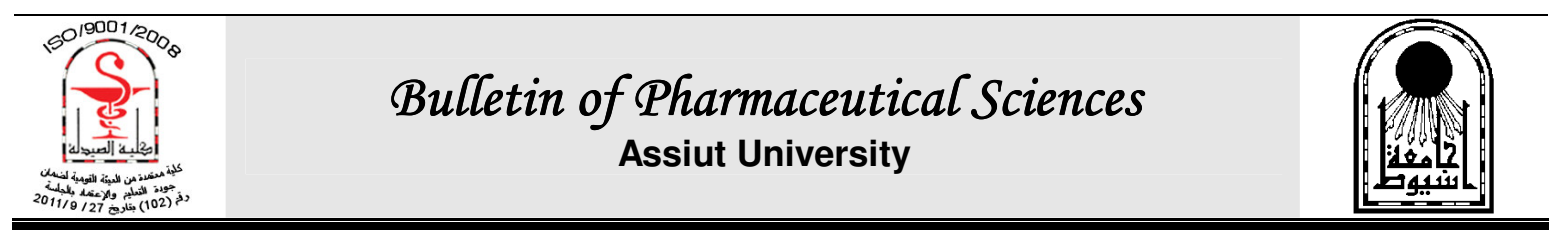

\title{
NIOSOMES AS AN ORAL DRUG DELIVERY SYSTEM CONTAINING TENOXICAM
}

\author{
Gamal Abdel Ghany Shazly ${ }^{1,2 *}$ \\ ${ }^{1}$ Department of Pharmaceutics, College of Pharmacy, King Saud University, P.O. Box 2457, \\ Riyadh 11451, Kingdom of Saudi Arabia \\ ${ }^{2}$ Department of Industrial Pharmacy, Faculty of Pharmacy, Assiut University, Assiut 71526, \\ Egypt
}

\begin{abstract}
Niosomes vesicles are microscopic lamellar structures formed by mixing with nonionic surfactant, cholesterol and phosphate in aqueous media. Using niosomes as drug delivery system affords several significant advantages over conventional drug therapy. The main aim of this study was to formulate suitable niosome-encapsulated drug delivery for tenoxicam (antiinflammatory drug) and estimate the percentage encapsulation efficiency, in-vitro release and in-vivo anti-inflammatory effect. Different non-ionic surfactants, cholesterol and different charge inducing agents were used in different molar ratios. Three different methods were used for niosomes preparation. The higher entrapment efficiency was observed with niosomes prepared from span 40, cholesterol and stearylamine in 45:45:10 molar ratios $\left(N_{11}\right)$. The invitro release study was found that niosomes exhibiting higher entrapment efficiency showed slower release rate of drug than other formulae. The results of in-vivo study revealed that the niosomes significantly enhanced the anti-inflammatory effect of tenoxicam. The present work concluded that tenoxicam loaded niosomes was effective in sustaining the drug release resulting in diminished side effects and improved patient compliance.
\end{abstract}

\section{INTRODUCTION}

The new trends in the design of drug delivery systems depends on the use of the ideas and methods of targeting drugs to specific sites in the body using various carriers and vehicles as drug delivery devices. Niosomes are colloidal particles formed from the selfassembly of non-ionic surfactants in aqueous medium resulting in closed bilayer structures $^{1 \& 2}$. Niosomes have been extensively considered for their potential to serve as carrier for delivery of drugs, antigens, hormones and other bioactive agents ${ }^{3}$. Formulation of a drug in niosomes can be expected to extend the presence of the drug in the systemic circulation and thus improve permeation in to target tissue and decrease the toxicity ${ }^{4}$. Niosomes have diverse advantages over normal dosage forms because they can act as drug reservoirs. Niosomes as drug carriers can play a progressively vital role in drug delivery ${ }^{5}$. Niosomes are similar to liposomes and act as drug carriers because they can incorporate both hydrophilic and lipophilic drugs. Niosomes have flexibility characteristics, including composition, fluidity and size, so they can be formulated according to the desired situation. Niosomes can increase oral bioavailability of poorly absorbed drugs and enhance skin penetration of many drugs ${ }^{6}$. Targeting the drug to the aimed tissues, sustaining its release and changing its pharmacokinetics can be performed using niosomes ${ }^{7}$. Formulating drugs in niosomes can reduce drug toxicity, enhance drug absorption, and hinder the elimination of the drug from the circulation due to slow drug release ${ }^{8}$.

Tenoxicam (TX) is a member of the oxicam group of non-steroidal antiinflammatory drugs (NSAIDs). TX, an enolic acid derivative, is nonselective COX-2

Received in 19/3/2015 \& Accepted in 22/6/2015 
inhibitor and so it has anti-inflammatory, analgesic, and antipyretic activity. Thus, TX is extensively used in the treatment of rheumatoid arthritis and osteoarthritis. TX is a poorly water soluble drug, so it has poor, erratic dissolution profile in gastrointestinal fluids, which consequently results in low and variable bioavailability ${ }^{9 \& 10}$.

Due to the decreased bioavailability and side effects associated with oral administration of TX, it is necessary to develop a novel drug delivery system of TX. So, the aim of this research work was to develop a suitable niosomal preparation of TX with an optimal encapsulation efficiency and drug release extended over a prolonged period with objectives of avoiding its most frequent side effect and gastric mucosal damage.

\section{MATERIALS AND METHODS}

Tenoxicam (TX), Span 20, 40, 60, 80, 85, cholesterol (Chol), Chloroform, dicetylphosphate (DCP) and stearylamine (SA) were purchased from Sigma Chemical Co., St. Louis, MO, USA and used without further purification. All ingredients were used as received.

\section{Methodology}

\section{Preparation of niosomes}

All TX $(2 \mathrm{mg} / \mathrm{ml})$ loaded niosomes were prepared from non-ionic surfactants, cholesterol, and with or without chargeinducing agents as stearylamine (SA) or dicetylphosphate (DCP) in different molar ratios (Table 1).

Table 1: Composition of niosomes containing TX $(2 \mathrm{mg} / \mathrm{ml})$.

\begin{tabular}{|c|c|c|c|c|c|c|c|c|c|}
\hline \multirow{2}{*}{$\begin{array}{c}\text { Formula } \\
\text { No. }\end{array}$} & \multicolumn{7}{|c|}{ Composition } & Preparation \\
\cline { 2 - 8 } & Span 20 & Span 40 & Span 60 & Span 80 & Span 85 & Chol & SA & DCP & method \\
\hline $\mathrm{N}_{1}$ & 50 & - & - & - & - & 50 & - & - & HS \\
$\mathrm{N}_{2}$ & - & 50 & - & - & - & 50 & - & - & HS \\
$\mathrm{N}_{3}$ & - & - & 50 & - & - & 50 & - & - & HS \\
$\mathrm{N}_{4}$ & - & - & - & 50 & - & 50 & - & - & HS \\
$\mathrm{N}_{5}$ & - & - & - & - & 50 & 50 & - & - & HS \\
$\mathrm{N}_{6}$ & - & 100 & - & - & - & - & - & - & HS \\
$\mathrm{N}_{7}$ & - & 90 & - & - & - & 10 & - & - & HS \\
$\mathrm{N}_{8}$ & - & 70 & - & - & - & 30 & - & - & HS \\
$\mathrm{N}_{9}$ & - & 48.5 & - & - & - & 48.5 & 3 & - & HS \\
$\mathrm{N}_{10}$ & - & 46.5 & - & - & - & 46.5 & 7 & - & HS \\
$\mathrm{N}_{11}$ & - & 45 & - & - & - & 45 & 10 & - & HS \\
$\mathrm{N}_{12}$ & - & 48.5 & - & - & - & 48.5 & - & 3 & HS \\
$\mathrm{N}_{13}$ & - & 46.5 & - & - & - & 46.5 & - & 7 & HS \\
$\mathrm{N}_{14}$ & - & 45 & - & - & - & 45 & - & 10 & HS \\
$\mathrm{N}_{15}$ & - & 50 & - & - & - & 50 & - & - & OSI \\
$\mathrm{N}_{16}$ & - & 50 & - & - & - & 50 & - & - & REP \\
$\mathrm{N}_{17}$ & - & 45 & - & - & - & 45 & - & 10 & OSI \\
$\mathrm{N}_{18}$ & - & 45 & - & - & - & 45 & - & 10 & REP \\
\hline \hline
\end{tabular}




\section{Hand-shaking method (HS)}

In a round-bottom flask, the lipid mixture and TX $(2 \mathrm{mg} / \mathrm{ml})$ were dissolved in $10 \mathrm{ml}$ chloroform. The organic solvent was evaporated at reduced pressure on a rotary evaporator at room temperature $\left(25^{\circ} \mathrm{C}\right)$ and 100 rpm until complete evaporation of the solvent was ensured and a dry smooth lipid film was deposited on the wall of the flask. The completely dried lipid film was hydrated with 5 $\mathrm{ml}$ of PBS (pH 7.4) at $60^{\circ} \mathrm{C}$ for one hour with intermittent shaking until the homogeneous milky white dispersion was formed ${ }^{11}$.

\section{Organic solvent-injection method (OSI)}

TX $(2 \mathrm{mg} / \mathrm{ml})$ and mixture of lipids were dissolved in $10 \mathrm{ml}$ chloroform and injected slowly through a needle at $0.25 \mathrm{ml} / \mathrm{min}$ in $5 \mathrm{ml}$ of PBS ( $\mathrm{pH} 7.4$ ) maintained at $60^{\circ} \mathrm{C}$. The organic solvent was allowed to be evaporated completely using a rotary evaporator. The produced vesicles are large unilamellar vesicles $(\text { LUVs })^{12}$.

\section{Reverse phase method (REP)}

$2 \mathrm{ml}$ of PBS (pH 7.4), was emulsified with the lipid mixture and TX $(2 \mathrm{mg} / \mathrm{ml})$ using a vortex mixer for $5 \mathrm{~min}$. The organic solvent was evaporated at room temperature until a thick gel. The obtained gel was mixed $3 \mathrm{ml}$ PBS ( $\mathrm{pH} 7.4$ ), with simultaneous vortexing. The evaporation was continued in a rotary evaporator until the hydration was completed ${ }^{13}$.

\section{Separation of free TX from niosomes}

The uncapsulated TX can be separated from niosomes by centrifugation ${ }^{14}$. Briefly, the prepared niosomes were diluted with PBS (10 $\mathrm{ml}$ ) and were then subjected to centrifugation (10,000 g for 30 minutes). The supernatant was then removed and the remaining niosomal pellets were diluted with PBS $(10 \mathrm{ml})$ and were subjected to centrifugation again as previous. These procedures were repeated three times. The obtained niosomes were then diluted with $4 \mathrm{ml}$ PBS for further investigation.

\section{Determination of TX entrapment efficiency}

A sample of $50 \mu \mathrm{l}$ of the prepared MLVs before removal of unencapsulated TX was taken and lysed with $0.5 \%(\mathrm{v} / \mathrm{v})$ Triton X-100 in distilled water (A). After removal of the unencapsulated TX by washing and centrifugation, $50 \mu \mathrm{l}$ were taken from the resuspended pellets and treated with triton $\mathrm{X}$ 100 as before (B). TX content was determined in both A and B by HPLC. The encapsulation efficiency (\%) was determined by the following equation:

$$
\% E E=\frac{A D_{b}}{A D a} \times 100
$$

Where, $\mathrm{ADa}$ and $\mathrm{ADb}$ are the amounts of $\mathrm{TX}$ in niosomes before and after the process of washing and centrifugation, respectively.

\section{HPLC analysis of TX}

TX amount was measured using a Waters HPLC system (Milford, MA, USA), equipped with a Dual Absorbance detector, a Binary HPLC pump, and a reversed-phase C18 column (4.6 $¥ 150 \mathrm{~mm}$, Hypersil, Asheville, NC, USA). The HPLC system was monitored by Empower (Waters) software. The mobile phase was a mixture of $0.02 \mathrm{M}$ disodium hydrogen phosphate and acetonitrile $(60: 40 \mathrm{v} / \mathrm{v})$, filtered through $0.45 \mathrm{~mm}$ membrane filter and eluted at a flow rate of $1.0 \mathrm{~mL} / \mathrm{min}$, injection volume, 20 $\mathrm{ml}$ and retention time $2.4 \mathrm{~min}$. Effluents were monitored at $344 \mathrm{~nm}^{15}$.

\section{In-vitro release of $\mathrm{TX}$}

Dialysis bag (Himedia dialysis membrane, 12,000-14,000 molecular weight cut-off) was used for carrying out the in-vitro release of the prepared niosomal TX. The dialysis membrane was wet in warm water for $10 \mathrm{~min}$, one end was sealed with a clip, the TX niosomes preparation solution $(2 \mathrm{ml}$ resuspended in $1 \mathrm{ml}$ PBS) was put into the bag and the bag was sealed with another closure clip to prevent leakage. The dialysis bag was placed immersed in $100 \mathrm{ml}$ of $\mathrm{PBS}, \mathrm{pH} 7.4$, at $37 \pm 2^{\circ} \mathrm{C}$. The medium was then subjected to stirring at 100 rpm. At a scheduled time, samples of medium $(2 \mathrm{ml})$ were withdrawn and substituted with fresh buffer and the amount of TX was determined using HPLC ${ }^{16}$.

\section{Transmission electron microscopy (TEM)}

The morphology of the prepared niosomal formulation was investigated using transmission electron microscopy (TEM). Briefly, a sufficient quantity of $1 \%$ phosphotungstic acid was gently mixed to an aliquot of 
the prepared niosomal formulation. A drop of the mixture was put on to the carbon-coated grid and the excess was then drained off. The grid was allowed to dry, and it was examined under TEM (Hitachi H7500, Japan) ${ }^{17}$.

\section{Evaluation of anti-inflammatory activity}

In-vivo anti-inflammatory activity of TX niosomes was assessed on the basis of the inhibition of the volume of the hind paw edema induced by injecting an irritant (formalin $1 \%$ $\mathrm{w} / \mathrm{v}$ in $0.9 \% \mathrm{w} / \mathrm{v}$ saline) into the rat's $\mathrm{paw}^{18}$. Formula $\mathrm{N}_{11}$ was selected as it showed the best encapsulation efficiency and the slowest invitro release among all formulations.

\section{Selection of animals}

Adult male Wistar Albino rats aging approximately 3 months ranging in weight from $150 \pm 10 \mathrm{~g}$ were obtained from the Animal Care Center, College of Pharmacy, King Saud University, Riyadh, Saudi Arabia. The animals were housed in metabolic cages under controlled environmental conditions $\left(25^{\circ} \mathrm{C}\right.$ and a $12 \mathrm{~h}$ light/dark cycle). Animals had free access to pulverized standard rat Pellet food and tap water. The protocol of this study has been followed the instruction of the Research Ethics Committee of College of Pharmacy, King Saud University, Riyadh, Saudi Arabia. The animals were divided into four groups, each consisting of five rats. The first group was considered as control without taking any medicament. The second group administered plain TX suspended with a few drops of propylene glycol orally using feeding cannula as a standard group in the dose equivalent to 2 $\mathrm{mg} / \mathrm{kg}$ body. The third and fourth groups received empty niosomes and niosomal suspension of TX $\left(\mathrm{N}_{11}\right)$ respectively in a dose of $2 \mathrm{mg} / \mathrm{kg}$ body weight ${ }^{19}$. After half an hour, the animals were generally anesthetized by intraperitoneal injection of $1 \mathrm{ml}$ of urethane (25\%). After one hour, $0.1 \mathrm{ml}$ formalin (10\%) was injected subcutaneously into the plantar region of the right hind paw for all groups. At time intervals $0,1,2,3,4$ and 6 hours, the inflammation was measured using 37140Plethysmometer (UgoBasileSrl., Comerio VA, Italy). The anti-inflammatory (\% response) was calculated according to the following equation:
Response $\%=(\mathrm{C}-\mathrm{T}) / \mathrm{C} * 100 \ldots \ldots \ldots \ldots . .(2)$

Where,

$\mathrm{C}=$ inflammation of right paw-inflammation of left paw for control rat

$\mathrm{T}=$ inflammation of right paw-inflammation of left paw for treated rat

\section{RESULTS AND DISCUSSION}

\section{Preparation of TX niosomes}

Niosomes containing TX were prepared using different types of surfactants, different concentration of surfactant, different concentration of Chol and two types of charge inducing agents (Table 1).

\section{Effect of surfactant type on the entrapment efficiency of TX in niosomes}

Figure 1 exhibits effect of surfactant type on \% $\mathrm{EE}$ of $\mathrm{TX}$ in niosomes (formulae. $\mathrm{N}_{1}$ $\mathrm{N}_{5}$ ). It was found that formula $\mathrm{N}_{2}$ showed the highest EE\%, followed by $\mathrm{N}_{3}, \mathrm{~N}_{1}, \mathrm{~N}_{5}$ and $\mathrm{N}_{4}$. This could be attributed to that formulae $\mathrm{N} 2$ and N3 contain span 40 and 60 respectively. Span 40 and 60 have the highest phase transition temperature $\left(\mathrm{T}_{\mathrm{C}}\right)$ of about $45^{\circ} \mathrm{C}$, $55^{\circ} \mathrm{C}$ respectively compared with other surfactants and so they are solid at room temperature. Thus, these surfactants produce niosomes with the highest $\% \mathrm{EE}^{20}$. These results are similar with those reported by Sathyavathi et l. $^{21}$, who found that Span 40 and 60 produce niosomes with higher entrapment efficiency of brimonidine tartrate than other spans.

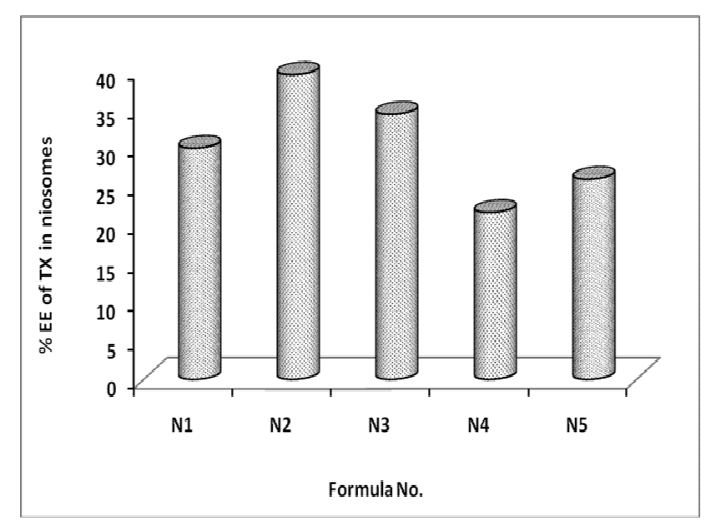

Fig. 1: Effect of surfactant type on \% EE of TX in niosomes. 
Effect of concentration of surfactant and Chol on the \% EE of TX in niosomes

Figure 2 shows effect of surfactant and Chol concentration on \% EE of TX in niosomes. From this figure, it was found that increasing the Chol content from $0 \%$ to $50 \%$ and decreasing surfactant content (formulae $\mathrm{N}_{6}$, $\mathrm{N}_{7}, \mathrm{~N}_{8}$ and $\mathrm{N}_{2}$ ) resulted in an increase in the \% EE of TX. This could be attributed to that an increase in Chol content leads to not only an increase in the vesicle size, but also making vesicle membrane more compact and organized. This finding is in accordance with that reported by Mokhtar, et $a l^{22}$, who found that a significant increase in flurbiprofen entrapment efficiency upon increasing Chol concentration.

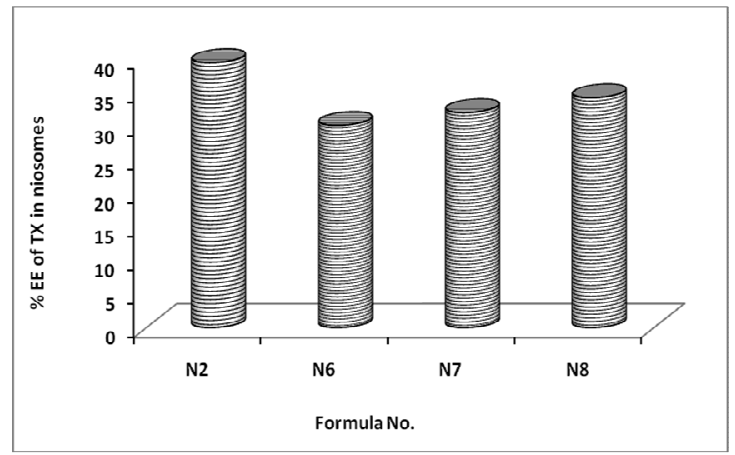

Fig. 2: Effect of surfactant and Chol concentration (molar ratio) on the \% $\mathrm{EE}$ of $\mathrm{TX}$ in niosomes.

\section{Effect of charge-inducing agents on the $\% \mathrm{EE}$ of TX in niosomes}

The effect of incorporation of chargeinducing agents on \% $\mathrm{EE}$ of TX in niosomes is presented in figure 3. As shown in this figure, it was obvious that positively charged niosomes showed the highest \% EE followed by neutral niosomes and negatively charged niosomes using the same span 40/Chol ratio. This could be attributed to strong interaction of the drug, which carries negative charge, with SA via formation of ion-pair forming lipophilic moiety which partitions into bilayers. This attraction resulted in higher \% EE of niosomes containing SA when compared to niosomes containing DCP and niosomes without chargeinducing agent. It was found, as the amount of charge-inducing agent increases, the \% $\mathrm{EE}$ increases ${ }^{23}$.

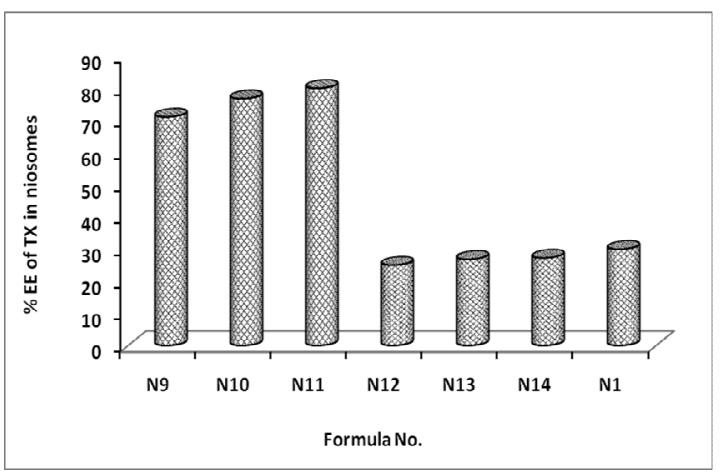

Fig. 3: Effect of charge-inducing agent on the $\%$ $\mathrm{EE}$ of TX in niosomes compared to neutral niosomes.

Effect of method of preparation on the entrapment efficiency of TX in niosomes

Figure 4 demonstrates the effect of the method of preparation on the \% $\mathrm{EE}$ of $\mathrm{TX}$ in niosomes. From the obtained results, it was found that the niosomes prepared by Handshaking Method exhibited the highest \% EE of TX among the other methods (organic solventinjection and reverse phase methods). This may be attributed to that the hand-shaking method lead to formation of niosomes higher number of lipid bilayers (MLVs) than the other methods and this exhibit the highest \% EE of $\mathrm{TX}^{24}$.

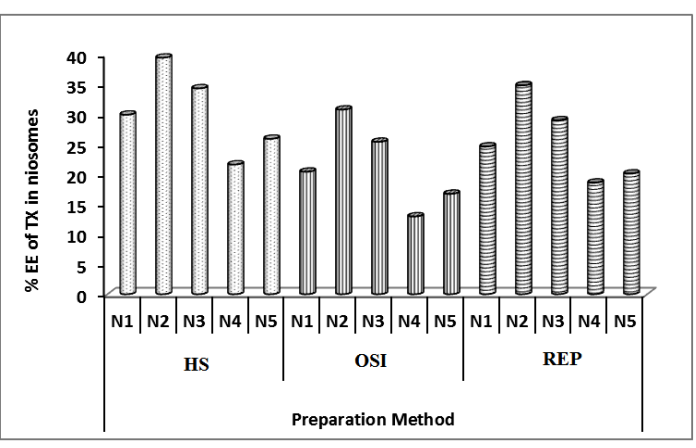

Fig. 4: Effect of method of preparation on the \% $\mathrm{EE}$ of TX in niosomes.

\section{Transmission electron microscopy}

TEM image of TX loaded niosomes was shown in figure 5 ( $a$ and $b$ ). As observed, the micrographs reveale that the prepared niosomes are spherical in shape and are bilayered structured in aggregate or in disperse collections. 


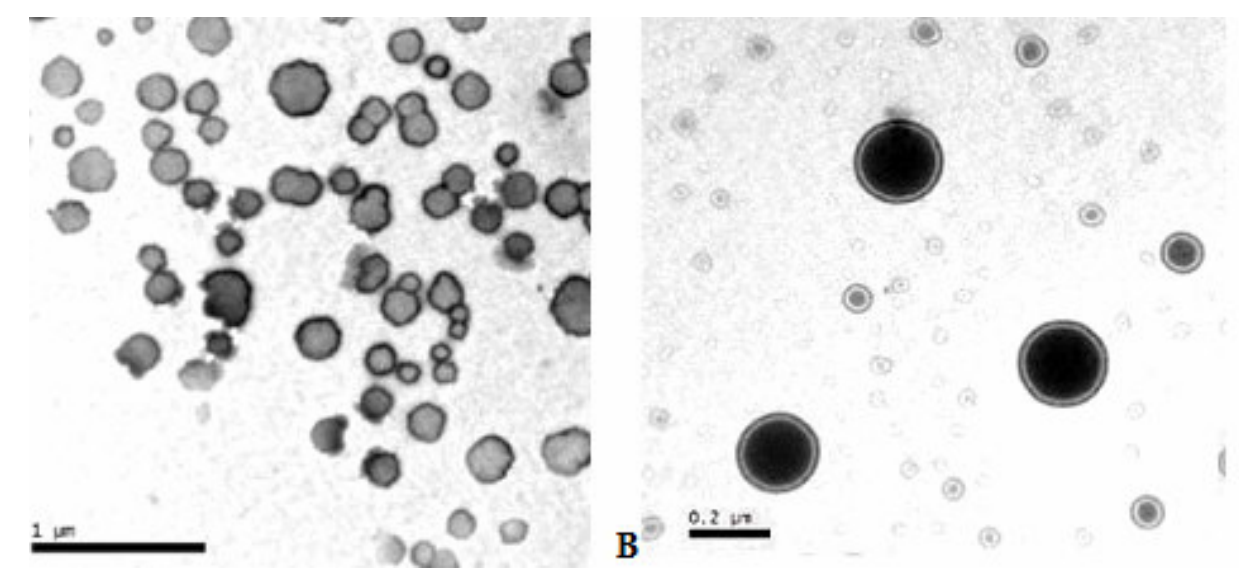

Fig. 5: TEM of TX niosomal suspensions of A: The molar ratio Span40: Chol (50:50) $\left(\mathrm{N}_{2}\right)$ and B: The molar ratio Span40: Chol: SA $(45: 45: 10)\left(\mathrm{N}_{11}\right)$.

\section{In-vitro release of TX from niosomes \\ Effect of lipid composition on the in-vitro release profiles of TX from niosomes}

Figure 6 demonstrated the in-vitro release profiles of TX from niosomes of different bilayer compositions. From these release profiles, it was found that incorporation of Chol (N2) reduced the percentage of drug released as compared to the formulation without chol (N6). This reduction in the TX release may be attributed to that chol has the ability to stabilize the bilayer vesicles producing more rigid and less permeable membrane $^{25}$. By adding a charged lipid e.g. SA to the formulation, a further reduction in TX release rate was observed. This further reduction of the drug release rate could be due to that inclusion of SA lead to squeeze the molecular packaging of the vesicle bilayers, and consequently reduced the drug release rate from charged niosomes. This result is similar with that reported by Mohamed et al. ${ }^{26}$, who found that the release of baclofen from niosomes was retarded by addition of chargeinducing agents.

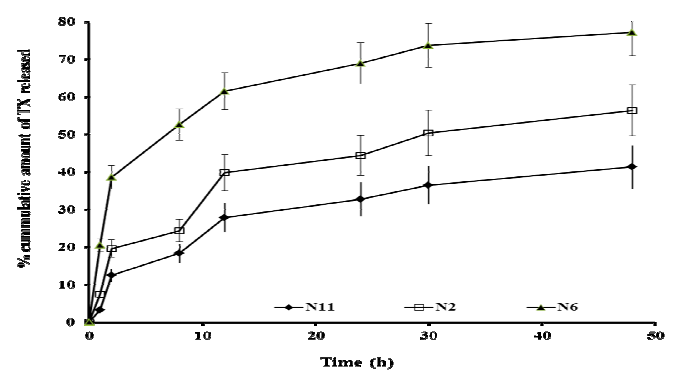

Fig. 6: Effect of lipid composition on the in-vitro release profiles of PRX from niosomes.

\section{Effect of surfactant structure on the release} rate of TX from niosomes

The effect of surfactant structure on the in-vitro release rate of TX from niosomes was illustrated in figure 7. Formulae $\mathrm{N}_{2}$ and $\mathrm{N}_{3}$ prepared using Span 40 and 60, respectively showed slower release rate compared to $\mathrm{N}_{1}, \mathrm{~N}_{4}$, and $\mathrm{N}_{5}$ prepared from Span 20, 80, and 85, respectively. This may be attributed to that span 40 and 60 possess higher phase transition temperature $\left(\mathrm{T}_{\mathrm{C}}\right)$, which is lead to the formation of more rigid less permeable bilayers than Span 20, 80, and 85 which form more permeable fluid bilayers ${ }^{27}$. The release rate of TX from $\mathrm{N}_{3}$ is slower than that from $\mathrm{N}_{2}$ and this may be due to that Span $60\left(\mathrm{~N}_{3}\right)$ having longer chain length than span $40\left(\mathrm{~N}_{2}\right)$ leading to more stable vesicles which gave delayed drug release. Span 60 plays an important role in the observed outcome due to the ordered gel state and higher phase transition temperature of Span $60^{28}$. Although span 80 and $85\left(\mathrm{~N}_{4}\right.$ and $\mathrm{N}_{5}$ respectively) have longer chain length than span 40 and $60\left(\mathrm{~N}_{2}\right.$ and $\mathrm{N}_{3}$ respectively), they gave higher release of drug as compared to that of span 40 and 60, as they have unsaturated alkyl chain, the double bond resulted in bending the chain. This made the adjacent molecules not to be tight when they form the membrane of noisome. This explains why the drug release is high. The same results were reported by Omar et al. ${ }^{29}$ who stated that the release of rosuvastatin calcium from span 80 was higher than Span 40 and 60. 


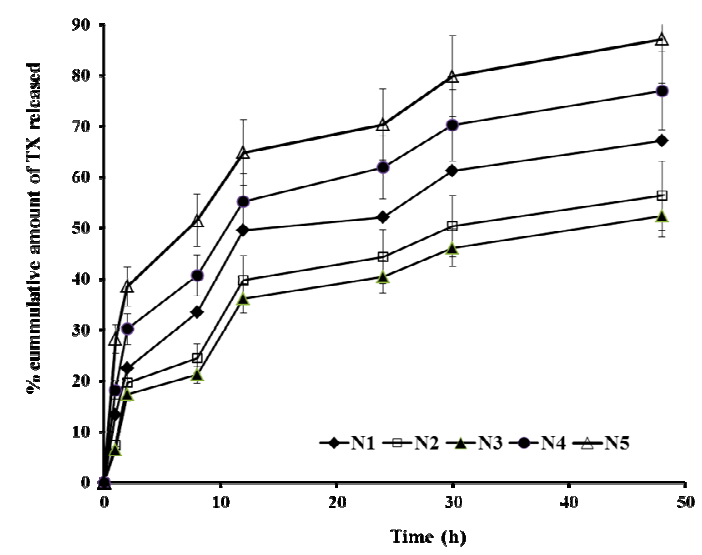

Fig. 7: Effect of surfactant structure on the release profiles of PRX from niosomes.

\section{Effect of chol concentration on the release rate of TX from niosomes}

Figure 8 illustrated the effect of chol concentration on the in-vitro release rate of TX from niosomes. The release pattern revealed that the rate of drug release was decreased by increasing cholesterol concentration, which may be due to intercalation of cholesterol in the bilayers. As the chol concentration increases, the hydrophobicity and stability of bilayer augmented and permeability diminished which results in powerfully trapping the hydrophobic drug into bilayers as vesicles formed. These results are similar to that reported by Abraham et al. $^{30}$, who found that the release of clobetasol propionate from niosomes decreased by increasing chol amount in the formulation.

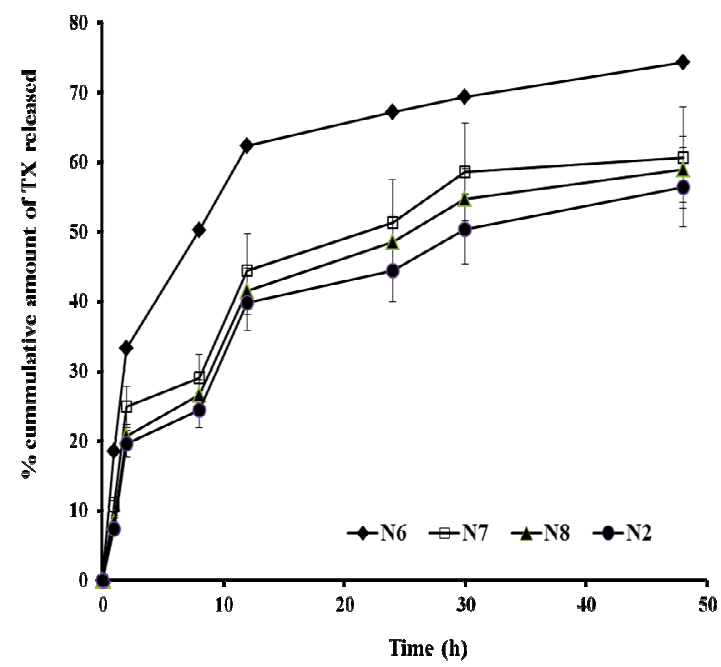

Fig. 8: Effect of cholesterol concentration on the release profiles of TX from niosomes.
Effect of type of charge-inducing agent on the release rate of $\mathrm{TX}$ from niosomes

The effect of inclusion of charge-inducing agent on the release of TX from niosomes was elucidated in figure 9. Upon comparing the amount of TX released from neutral niosomes $\left(\mathrm{N}_{2}\right)$, positively charged niosomes of different molar ratio $\left(\mathrm{N}_{9}, \mathrm{~N}_{10}\right.$ and $\left.\mathrm{N}_{11}\right)$ and that negatively charged niosomes of different molar ratio $\left(\mathrm{N}_{12}, \mathrm{~N}_{13}\right.$ and $\left.\mathrm{N}_{14}\right)$, it can be decided that the inclusion of charge inducing agents (either positive or negative) reduced the amount of TX released at all times of investigation. These results may be contributed to that the charge inducing agents resulted in stabilizing the niosomal membrane structure, rendering it less permeable $^{31}$.

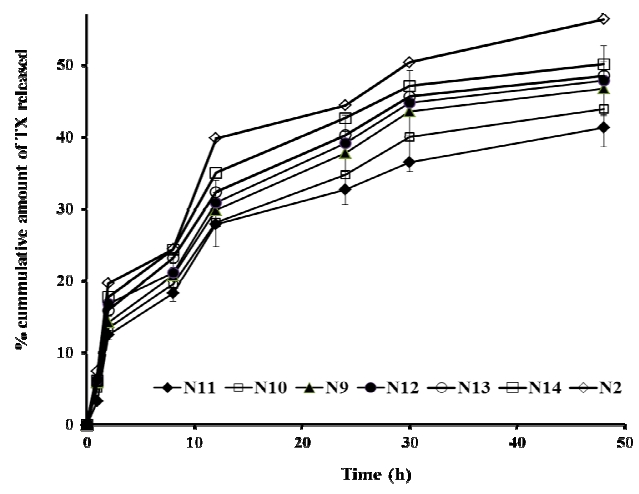

Fig. 9: Effect of type of charge-inducing agents and their concentration on the release profiles of TX from niosomes.

\section{Effect of method of preparation on the release rate of $\mathrm{TX}$ from niosomes}

Figure 10 reveals the effect of preparation method on the release rate of TX from niosomes. From this figure, it was concluded that the release rate of TX from niosomes prepared by the OSI method is higher than REP and HS methods respectively. This may be attributed to the type of vesicles prepared by each method. The OSI method produced unilamellar vesicles and HS and REP methods produced multilamellar vesicles. Unilamellar vesicles posse a large surface area to volume ratio and have only a single lipid bilayer barrier to drug diffusion as compared to the multilamellar vesicles, which composed of several lipid bilayers as concentric spheres separated by aqueous compartments. These results are in accordance with those described by Chawda et $a l^{24}$, who revealed that the release of nimesulide from niosomes was 
higher from unilamellar vesicles than multilamellar vesicles.

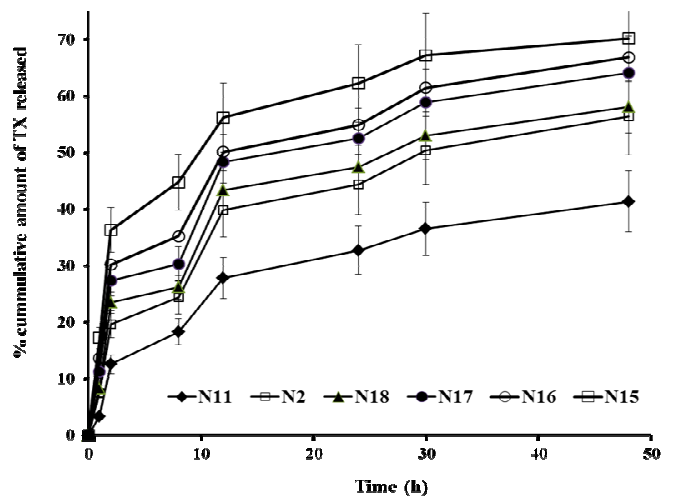

Fig. 10: Effect of preparation method on the release profiles of TX from niosomes.

\section{In-vivo anti-inflammatory study}

The percent of the edema inhibition due to TX in both plain TX and TX entrapped niosomes was shown in figure 11. It is obvious that TX suspension and $\mathrm{N}_{11}$ significantly reduced the inflammation compared to the control. The percent response of formula $\mathrm{N}_{11}$ was higher as compared with that of TX suspension. The magnitude of inhibition was more than $80 \%$ after 6 hours. Similar results were obtained by Sammour et $a l^{32}$, who observed that the anti-inflammatory activity of piroxicam-entrapped liposomes is greater than that obtained by piroxicam in the free form. The duration of anti-inflammatory activity of $\mathrm{N}_{11}$ noisome was long and tis may be attributed to the sustained release of the entrapped TX. This confirms the sustained drug release of niosomes $^{33}$. It was also observed that empty niosomes could not inhibit the oedema formation significantly $(\mathrm{P}<0.05)^{34}$.

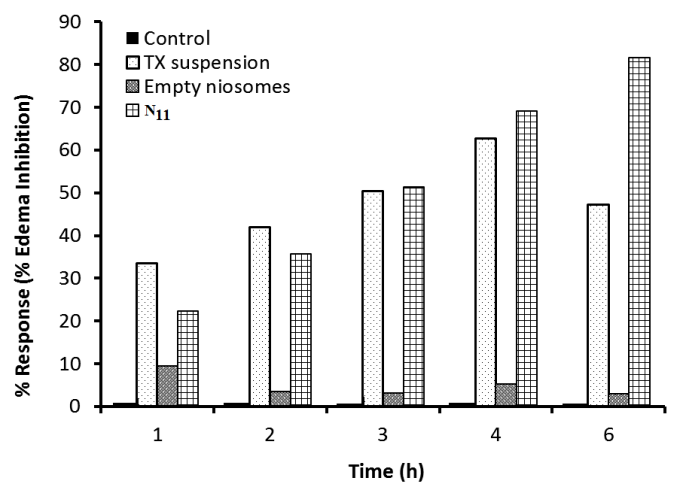

Fig. 11: Percent Edema inhibition after oral administration of different formulations of TX.

\section{Conclusion}

Different niosomal formulations containing TX were prepared using different surfactants, charge inducing agents and different preparation methods. From the data obtained, it can be concluded that \% EE of TX into niosomes depends on surfactant type, chol content, type and amount of charge inducing agents, and method of preparation. The drug release of TX from niosomes was found to be affected also by above mentioned variables. The niosomal delivery system considerably enhanced the anti-inflammatory of TX.

\section{Acknowledgments}

The Author extends his appreciation to the Deanship of Scientific Research at King Saud University for funding the work through the research group project No. RGP-VPP-139.

\section{REFERENCES}

1- R. Mujoria and R. Bodlab, "NiosomesChallenge in preparation for pharmaceutical Scientist", Int. J. App. Pharm., 3 (3), 11-15 (2011).

2- G. Kumarn and P. Rajeshwarrao, "Nonionic surfactant vesicular systems for effective drug delivery-an overview", Acta. Pharmaceutica Sinica B, 1 (4), 208-219 (2011).

3- M. Malhotra and N. K Jain, "Niosomes as drug carriers", Indian Drugs, 31 (3), 8186 (1994).

4- B. Arul, "The effect of niosomes on the kinetics of ketorolac tromethamine", The Eastern Pharmacist, November, 115-117 (1998).

5- R. Arora and C. P. Jain, "Advances in niosomes as a drug carrier: A review", Asian J. Pharmaceuticals, 1 (1), 29-39 (2007).

6- S. Srinivas, "Preparation and Evaluation of Niosomes Containing Aceclofenac Sodium", M. Thesis, Karnataka, Bangalore, Raichur (2010).

7- G. N. Devaraj, S. R. Parakh, R. Devraj, S. S. Apte, B. R. Rao and D. Rambhau, "Release studies on niosomes containing fatty alcohols as bilayer stabilizers instead of cholesterol", J. Coll. Interf. Sci., 251, 360-365 (2002). 
8- A. Manosroi, P. Wongtrakul, J. Manosroi, H. Sakai, F. Sugawara, M. Yuasa and M. Abe, "Characterization of vesicles prepared with various non-ionic surfactants mixed with cholesterol", Colloids and Surfaces B, Biointerfaces, 30, 129-138 (2003).

9- V. Vikranth, K. S. Pankaj, K. Poonam, K. Manali and S. Manali, "Physico chemical characterization of solid dispersion system of tadalfil with poloxamer 407", Acta. Pharm., 59, 453-461 (2009).

10- N. Madhuri, H. B. krishna, H. O. Dong, R. K. Young, H. S. Joon and O. K. Jong, "Enhanced dissolution of ibuprofen using solid dispersion with poloxamer 407", Arch. Pharm. Res., 31 (11), 1497-1507 (2008).

11- R. Parthibarajan, S. Pradeep Kumar, N. L. Gowri, L. Balakishan, B. Srinivas, V. Bhagya A. Radha and K. Sheshma, "Design and in-vitro evaluation of voriconazole niosomes", J. Pharm. Pharm. Sci., 5 (3), 604-611 (2013).

12- M. E. Yildiz, R. K. Prud'homme, I. Robb and D. H. Adamson, "Formation and characterization of polymersomes made by a solvent injection method", Polymers for Advanced Technologies, 18 (6), 427-432 (2007).

13- M. Zarei, D. Norouzian, B. Honarvar, M. Mohammadi and H. F. Shamabadi, "Paclitaxel loaded niosome nanoparticle formulation prepared via reverse phase evaporation method: An in-vitro evaluation", Pak. J. Biol. Sci., 16 (6), 295-8 (2013).

14- G. Shazly, "Design and evaluation of sustained released liposomal preparations containing adenosine monophosphate", Digest Journal of Nanomaterials and Biostructures, 7 (4), 1679-1687 (2012).

15- H. Mohammad, H. Semreen and Y. Hassan, "LC-UV method development and validation for the non-steroidal antiinflammatory agent tenoxicam", Journal of Liquid Chromatography \& Related Technologies, 33 (5), 720-729 (2010).

16- R. Kandasamy and S. Veintramuthu, "Formulation and optimization of zidovudine niosomes", AAPS Pharm. Sci. Tech., 11 (3), 1119-1127 (2010).
17- S. R. Mohamed, A. B. Alia, M. Marwa and M. Amira, "Niosomes as a novel pharmaceutical formulation encapsulating the hepatoprotective drug silymarin", Int. J. Pharm. Pharmac. Sci., 4 (1), 549-559 (2012).

18- G. Shazly, M. Badran, K. Zoheir and A. Alomrani, "Utilization of spray drying technique for improvement of dissolution and anti-inflammatory effect of Meloxicam", Pak. J. Pharm. Sci., 28 (1), 103-111 (2015).

19- T. Hakan, H. Z. Toklu, N. Biber, H. Celik, C. Erzik, A. V. Oğünç, S. Çetinel S and G. Sener, "Meloxicam exerts neuroprotection on spinal cord trauma in rats", Int. J. Neurosci., 121 (3), 142-148 (2011).

20- G. Madhu, V. Bhuvaneshwar, M. Neeraj and P. Suresh, "Effect of Surfactants on the Characteristics of Fluconazole Niosomes for Enhanced Cutaneous Delivery", Artif. Cells Blood Substit. Immobil. Biotechnol., 39 (6), 376-84 (2011).

21- V. Sathyavathi, A. A. hasansathali, R. Ilavarasan and T. Sangeetha, "Formulation and evaluation of niosomal in situ gel Ocular delivery system of brimonidine tartrate", Int. J. Science Pharma. Res., 2 (1), 82-95 (2012).

22- M. Mahmoud, A. Omaima, M. Sammour, A. Mohammed and N. Hammad, "Effect of some formulation parameters on flurbiprofen encapsulation and release rates of niosomes prepared from proniosomes", Int. J. Pharm., 361, 104111 (2008).

23- B. J. Varaporn, V. Teeranachaideekul and T. Supaperm, "Effect of charged and nonionic membrane additives on physicochemical properties and stability of niosomes", AAPS Pharm. Sci. Tech., 9 (3), 851-895 (2008).

24- H. S. Chawda, C .P. Jain and B .N. Kumar, "Formulation, characterization, stability and in-vitro evaluation of nimesulide Niosomes". Pharmacophore, 2 (3), 168-185 (2011).

25- A. Hamdy, F. Usama, and M. Hossam, "Effects of surfactant type and cholesterol level on niosomes physical properties and in-vivo ocular performance using timolol 
maleate as a model drug", J. Pharm. Invest., 44 (5), 329-337 (2014).

26- A. Mohamed, R. Ehab, S. Mohamed, A. Gehad and M. Samar, "Formulation and evaluation of topical niosomal gel of baclofen", J. Chem. Pharm. Res., 7 (1), 277-288 (2015).

27- P. Khazaeli and A. Pardakhty, "Caffeineloaded niosomes: Characterization and invitro release studies", Drug Delivery, 14, 447-452 (2007).

28- A. Ismail, A. Sanaa, A. Medhat and M. Ahmed M, "Influence of a niosomal formulation on the oral bioavailability of acyclovir in rabbits", AAPS Pharm. Sci. Tech., 8 (4), Article 106 (2007).

29- S. Omar, H. Laith and K. Wedad, "Formulation and in-vitro Evaluation of rosuvastatin calcium niosomes", Int. J. Pharm. Pharm. Sci., 5 (4), 525-535 (2013).

30- M. Abraham, H. Abdul, R. Vijaya and A. Gokila, "Formulation and evaluation of topical drug delivery system containing clobetasol propionate niosomes", $\mathbf{S c i}$. Revs. Chem. Commun., 1 (1), 7-17 (2011).
31- G. Abdelbary and N. El-gendy, "Niosomeencapsulated gentamicin for ophthalmic controlled delivery", AAPS Pharm. Sci. Tech., 9 Suppl. 3, 740-7 (2008).

32- O. A. Sammour, H. H. Al-Zuhair and M. I. El-Sayed,"Inhibitory effect of liposomeencapsulated piroxicam on inflammation and gastric mucosal damage", Pharm. Ind., 60 (12), 1084-1087 (1998).

33- A. J. Baillie, A. T. Florence, L. R. Hume, G. T. Muirhead and A. Rogerson, "The Preparation and properties of niosomesnon-ionic surfactant vesicles", J. Pharm. Pharmcol., 37, 863-868 (1985).

34- Y. Raji, U. S. UdohS, O. O. Oluwadara, O. S. Akinsomosoye, O. Awobajo and K. Adeshoga, "Anti-inflammatory and analgesic properties of the rhizome extract of zingiber officinale", Afr. J. Biomed. Res., 5,121-124 (2002). 


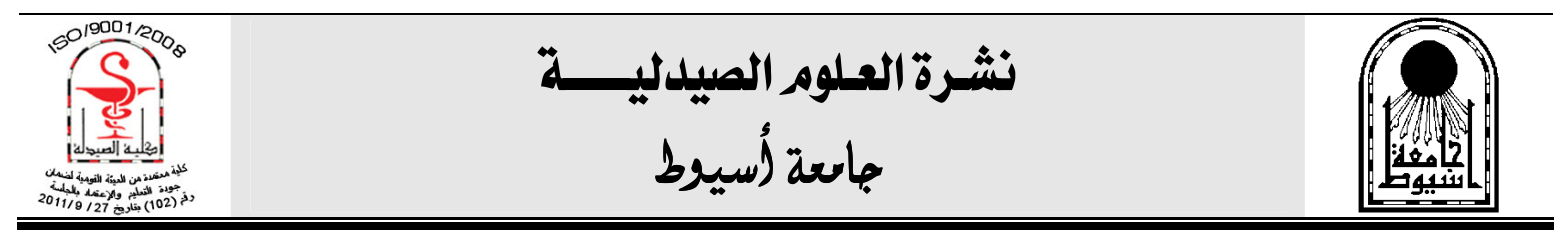

\section{النيوزومات كنظام لانطلاق الدواء عن طريق الفم يحتوى على عقار التينوكسام

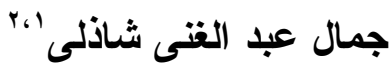

\section{'قسم الصيدلانيات ، كليـة الصيدلة ، جامعـة الملك سعود ، ص.ب. بهع ، الريـاض

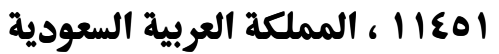

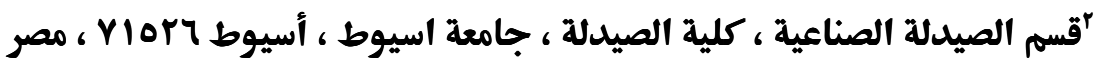

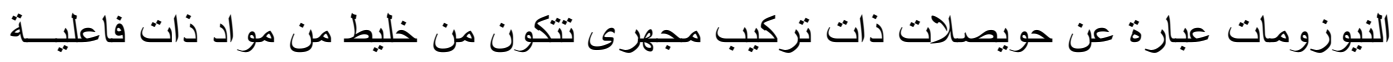

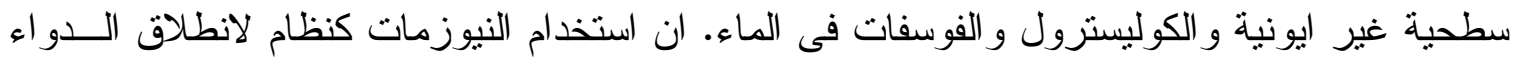

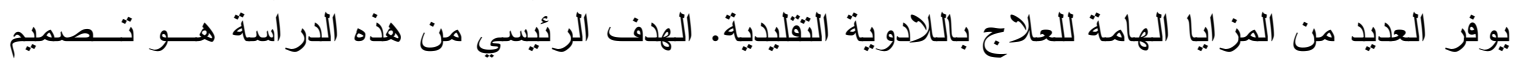

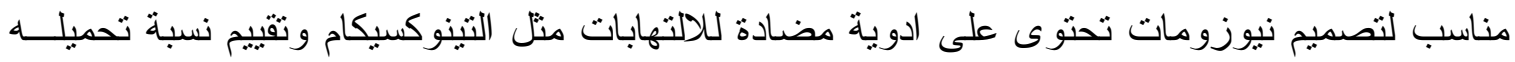

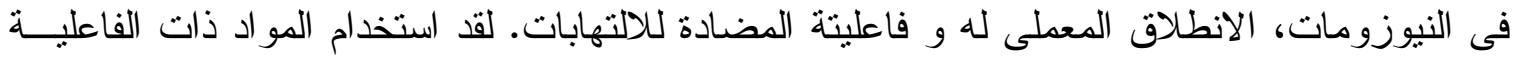

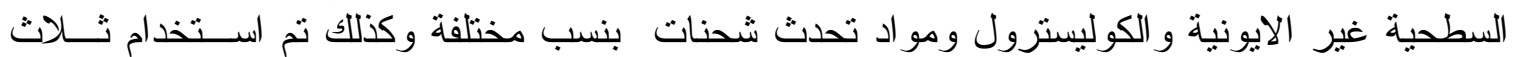

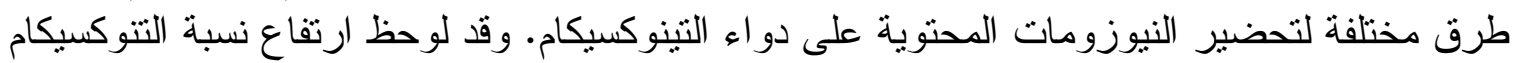

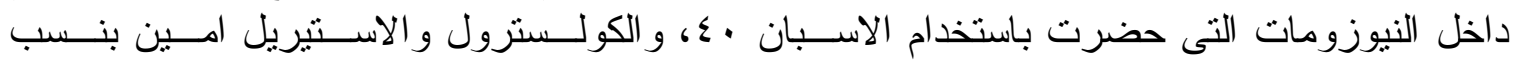

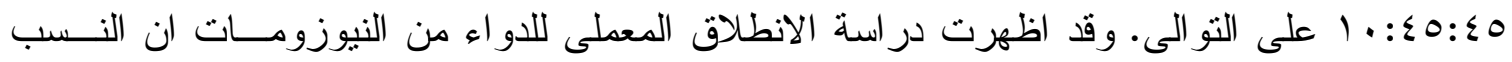

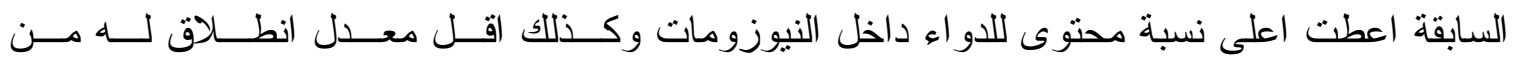

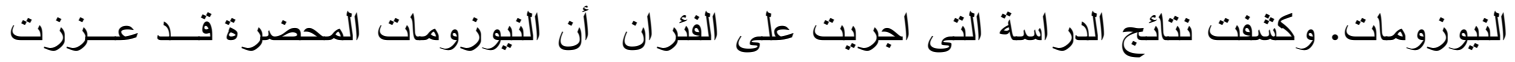

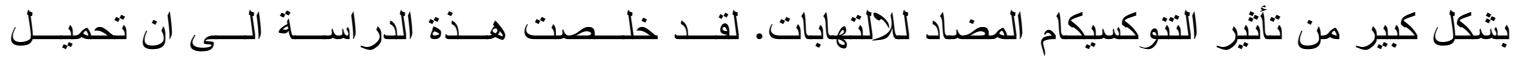

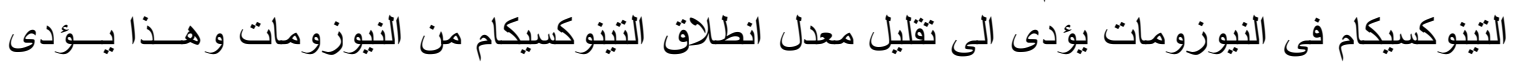

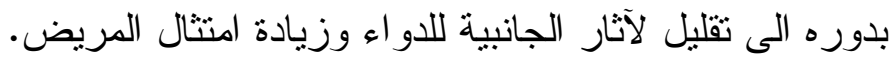

